This is the version of the article accepted for publication in Orientalistische Literaturzeitung published by de Gruyter https://www.degruyter.com/ https://doi.org/10.1515/olzg-2016-0022

Accepted version downloaded from SOAS Research Online: http://eprints.soas.ac.uk/26563

\title{
Lämmerhirt, Kai: Die sumerische Königshymne Šulgi F
}

Bei dem im Folgenden zu besprechenden Werk handelt es sich um die editio princeps der sumerischen Königshymne Šulgi F. Es ergänzt in vorbildlicher Weise die von Adam Falkenstein und insbesondere von Jacob Klein mit seinen Editionen von Šulgi A, D und X (Three Šulgi Hymns: Sumerian Royal Hymns Glorifying King Šulgi of Ur, Ramat Gan 1981) begonnene Erschließung des Corpus dieser Preislieder. Bedenkt man, dass Šulgi (2094-2047 BCE) mit einer Regierungszeit von bemerkenswerten 48 Jahren als der wichtigste und berühmteste Herrscher der dritten Dynastie von Ur gelten kann, der schon zu Lebzeiten vergöttlicht wurde und im kulturellen Gedächtnis über Jahrhunderte präsent blieb, mag es erstaunen, dass noch nicht alle ihm gewidmeten Hymnen in umfassenden Editionen vorgelegt wurden. Daher stellt die vorliegende Monographie einen umso willkommeneren Beitrag darund das nicht zuletzt in Hinblick auf die Tatsache, dass Šulgi F mit ursprünglich über 300 Zeilen zu den umfangreichsten Hymnen dieses Königs zählt.

Die Arbeit, welche eine überarbeitete Fassung der an der Universität Jena eingereichten Magisterarbeit des Vf. darstellt, ist in traditioneller Weise in eine Einleitung (I), eine ausführliche Beschreibung der Textzeugen inklusive Rekonstruktionsversuchen (II) sowie die eigentliche, aus Umschrift, Übersetzung und Zeilenkommentar bestehende Textedition (III-V) gegliedert. Abgerundet wird sie durch ein Verzeichnis der Editionen und der Textausgaben besprochener Werke (VI), ein Abkürzungsverzeichnis, Angaben zur Zitierweise und die Bibliographie (VII), Indices zu in Šulgi F bezeugten Personen, Orten, Lemmata und zitierten Textstellen mit Übersetzung (VIII) sowie Abbildungen, die eine neue Kopie des Hauptmanuskriptes durch den Vf., Kollationsergebnisse, Größenvergleiche bestimmter Tafeln und Fotos des Hauptmanuskriptes sowie der unpublizierten Tafeln aus dem University Museum in Philadelphia umfassen (IX).

Die Einleitung beginnt mit knappen Angaben zu Länge und Erhaltungszustand von Šulgi F sowie der generellen Einordnung in das Corpus der Šulgi-Hymnen (§1). So sei zwar von einer rekonstruierten Gesamtlänge von über 300 Zeilen auszugehen, weswegen Šulgi F mit Šulgi B (385 Z.), C (> 270 Z.), D (> 397 Z.) und E (257 Z.) vergleichbar sei, allerdings ist nur das erste Drittel der Hymne (Z. 1-97) weitestgehend erhalten; der Rest (Segmente a-e) bleibt 
fragmentarisch. Die nicht weiter erläuterte Feststellung, dass sich Šulgi F im Gegensatz zu den eben genannten Preisliedern nicht einer bestimmten Subgattung der Königshymnen zuordnen lasse, sondern eine Mittelstellung zwischen Selbstlobhymnus (wie B, C, E) und Königsepos (wie D) einnehme, leitet zu einer tabellarischen Klassifizierung der ŠulgiHymnen (§2) über. Unterschieden wird in Typ I (Götterhymne mit Nennung des Königs), zu der die Mehrzahl der Šulgi-Hymnen gerechnet wird, Typ II (Stilisierung des Königs in der 2./3. Ps.), Typ III (Stilisierung des Königs in der 1. Ps.) und Typ IV (Königsepos).

Stellenweise hätte man sich Erläuterungen der Typisierung gewünscht. So bleibt z.B. unklar, warum Vf. im Gegensatz zu dem in Anm. 4 zitierten G. Frayne Šulgi I nicht zu Šulgi H (Typ I) stellt, sondern unter Typ II einordnet. Leider wird im Folgenden ebenso wenig ausgeführt, welche Passagen von Šulgi B welchem Typ zuzuordnen sind und wie sie sich zueinander verhalten. Ein kurzgefasstes Strukturschema, komplementär zur detaillierten Inhaltsangabe in $\S 4$, würde übersichtlich die Einteilung in ,epischen’ Beginn und abschließendes Selbstlob ergeben:

1-29 Prolog

mit einem Katalog der Aufgaben des Herrschers bzw. dem Zweck des Königtums im Allgemeinen sowie der Erzeugung Šulgis als idealem Herrscher im

Besonderen (Refrain 1)

30-54 Narrative I

Geschehnisse in der Vorzeit: (positiver) Schicksalsentscheid für ganz Sumer durch An; Befriedung der äußeren Feinde durch Utu; Überfluss im Land durch die Himmelsgöttin und Utu

55-58 Erster eingeschobener Selbstlob-Refrain Šulgis

59-97 Narrative II

Šulgis Berufung zum Königtum, individueller Schicksalsentscheid für

Šulgi durch Utu; Bestätigung durch Enlil dank Nannas/Dilimbabbars Vermittlung

Beginn des Selbstlobs (vgl. E ii 12'f.)

[Segmente a und b],

c Wohlwollen der Götter und göttliche Abkunft Šulgis

d Von Überfluss geprägte Regierungszeit und überlegene Kriegskunst

e.5-30 Von Überfluss geprägte Regierungszeit

e.33-56 Šulgi als furchteinflößender (,stoßender“) Krieger 
e.59-69 Verschriftung der Hymne (mit Hilfe?) von Nissaba

e.70 Doxologie an Dilimbabbar

Auf die Klassifizierung der Hymne innerhalb des Corpus der Šulgi-Hymnen folgt ein Abriss der Forschungsgeschichte ( $\$ 3)$, der mit dem Bekanntwerden des in Jena befindlichen Hauptmanuskriptes A (HS 1460) durch I. Bernhardt und S.N. Kramer 1955/6 beginnt, und mit der jüngsten Identifikation der neuen Textzeugen F, G und g aus Philadelphia durch J. Klein, J. Peterson und S. Tinney endet.

In $§ 4$ der Einleitung präsentiert Vf. eine detaillierte Inhaltsangabe, die er nach Sinneinheiten und Textsegmenten gliedert. Anders als die bescheidene Überschrift „Inhaltsangabe“ suggeriert, handelt es sich hierbei jedoch nicht nur um ein einfaches Resümee des Textes. Vielmehr zieht Vf. zahlreiche Zitate aus anderen sumerischen Literaturwerken zum Vergleich und zur Illustration bestimmter Motive heran. Dies hilft nicht nur bei der Kontextualisierung einzelner Textpassagen, sondern stellt auch des Vf. solide Kenntnis der Materie unter Beweis.

Der erste Textabschnitt, Z. 1-19, beinhaltet einen Aufgabenkatalog für den Herrscher, welcher die materielle Versorgung des Landes sowie das Abwehren von Feinden, das Darbringen der Erstlingsgaben und die Sorge für Gerechtigkeit umfasst. Besonders ausführlich widmet sich Vf. hier dem nisa $\hat{g}$-Opfer. Da „literarische bzw. inschriftliche Bezugnahmen auf das in Nippur dargebrachte nisag-Opfer vergleichsweise selten zu finden“ seien (S. 6), verweist Vf. auf die ausführlichere Schilderung der Gaben im Mythos „Nannas Fahrt nach Nippur“ und fügt eine diachron angeordnete Übersicht über alle weiteren bekannten Belege aus Königsinschriften und literarischen Quellen an (S. 7-9). Tatsächlich ist die vom Vf. hervorgehobene Diskrepanz zwischen spärlichen literarischen Zeugnissen gegenüber zahlreichen Belegen aus Wirtschaftsurkunden erstaunlich: obwohl über 40 Hymnen auf Ur III-zeitliche Herrscher bekannt sind, wird das nis a $\hat{g}$-Opfer laut Aussage des Vf. nur in Šulgi F wörtlich erwähnt (S. 6). Hier wäre jedoch ein Verweis auf Šulgi N 91 anzufügen gewesen, wenngleich der Text auch vom Vf. auf S. 95 mit Anm. 5 zu Recht aus dem hymnischen Corpus ausgeschlossen wurde (,Schlaflied' für einen Sohn Šulgis). Dort heißt es (in fragmentarischem Kontext): [peš]-tur-zi nisaĝ-bi-a mu-un-de 6 „Er hat [Dattel]palmenschösslinge als Teil der Erstlingsgaben gebracht.“ 
Daraufhin (S. 9-10) wird die königliche Sorge um Gerechtigkeit besprochen, welche der Text in einem ungewöhnlichen literarischen Bild präsentiert: Das Böse (ni $\hat{g}_{2}-\mathrm{NE}$.RU) wird als „kein schwerer Stein“ (na4 mah nu-me-a) beschrieben, wofür Vf. zwei mögliche Interpretationen vorschlägt. Entweder es handele sich um ein sonst unbekanntes Sprichwort mit der Aussage, dass ,das Böse nichts ist, was man einmal in den en gur wirft und das dann nie wieder an die Oberfläche zurückkommt“ (S. 9), sondern stetig unten festgehalten, d.h. bekämpft werden muss. Alternativ könne nu-me-a einen irrealen Komparativsatz bilden: „um das Böse, wie wenn es ein großer Stein wäre, im engur festzuhalten“ (S. 10). Beide Interpretationsmöglichkeiten finden sich auch in der Übersetzung wieder. Jedoch ist einschränkend zu bemerken, dass das $\mathrm{na}_{4}$ in beiden erhaltenen Manuskripten epigraphisch unsicher und „festhalten“ für tab (Ursprungsbedeutung wohl „Seite an Seite sein“) wie auch „schwer“ für mah (zu erwarten wäre eher dugud) recht freie Übersetzungen sind.

Im zweiten Textabschnitt (Z. 30-58) wird das Verhältnis Šulgis zum Himmelsgott An, zum Sonnengott Utu und zur Himmelsgöttin (An) beschrieben. Besonderes Augenmerk wird jedoch auf den Šulgi selbst in den Mund gelegten Refrain (Z. 55-56) gelegt, in welchem die Göttin Ĝeštinana laut Vf. als „Autorin des Liedes“ (Hervorhebung der Rez.) erwähnt werde (S. 11). Zur Erläuterung ihrer Funktion zieht er zwei Passagen aus anderen Literaturwerken heran. So ist Ĝeštinana in Šulgi E (Z. 14-22) die Auftraggeberin, welche die Gelehrten auffordert, Lieder auf den Herrscher zu komponieren, während Dumuzi seine Schwester in „Dumuzis Traum“ (Z. 21f.) als „Sängerin, die um alle Lieder weiß“, preist. Bedenkt man den fragmentarischen Schluss von Šulgi F, wo die Schreibergöttin Nissaba in Zusammenhang mit Lapislazulitafel und Schreibrohr genannt wird, so erscheint es aus Sicht der Rez. plausibler, in Ĝeštinana lediglich die Sängerin, nicht aber unbedingt die Autorin der Hymne zu sehen. Dies würde auch gut zu dem Epitheton aus „Dumuzis Traum“ passen, zumal Ĝeštinana in mehreren Hymnen als Šulgis göttliche Schwester vorgestellt wird, was Šulgi indirekt mit Dumuzi gleichsetzt. Oder basiert Vf. diese Interpretation implizit auf dem in $§ 5$ (s.u.) vorgeschlagenen Traditionsmodell, nach welchem die Šulgi-Hymnen zunächst von Sängern mündlich weitergegeben wurden, bevor sie einige Jahrhunderte später ihre schriftliche Form fanden?

Abschließend wird die Reihenfolge der genannten Götter An - Utu - Nanna, einer Idee von Wilcke folgend, als mögliche „mythopoetisch ausgeformte“ Krönungsreise von Uruk über Larsa nach Ur, der Reichshauptstadt, interpretiert (S. 12-13). Einschränkend hätte hier jedoch 
schon auf in $§ 5$ vorgetragene Skepsis an der Verwendung literarischer Texte für die historische Rekonstruktion verwiesen werden können; man gewinnt den Eindruck, dass gelegentlich verschiedene Stadien der Magisterarbeit nichtharmonisiert nebeneinander stehengeblieben sind. Es verwundert ferner, dass sich im Kontext dieses Abschnitts kein Verweis auf R.H. Wilkinson, Mesopotamian Coronation and Accession Rites in the NeoSumerian and Early Old-Babylonian Periods, c. 2100-1800 B.C., Univ.-Diss. Minnesota, 1986, findet.

Während die Segmente a und b für ein Verständnis zu fragmentarisch sind, beinhalten die Segmente c und d neben der Investitur Šulgis und der Verleihung eines Thronnamens durch Ninhursaĝa hauptsächlich bereits Bekanntes. Im letzten Textsegment (e) hingegen rühmt sich Šulgis eindrucksvoll seiner Kampfeskraft, wobei fast jede Zeile in einem „stakkatohafte[n]“ (S. 16) $d u_{7}\left(-d u_{7}-m_{e}-e_{3}\right)$, ich bin es, der stößt“ endet. Das nur fragmentarisch erhaltene Ende der Hymne interpretiert Vf. unter Verweis auf Šulgi B 311-3 und E 249-51 dahingehend, dass „die Schreibergöttin Nisaba Ĝeštinanas Lied auf einer Tafel festhalten soll, damit es den Sängern bekannt gemacht werden kann“(S. 16f.). Die hier postulierte Verschriftung der Hymne als Voraussetzung für die Darbietung durch Sänger widerspricht dabei dem im nächsten Paragraphen vorgeschlagenen Überlieferungsmodell (s. auch schon oben).

Im methodisch sehr umsichtigen $§ 5$ widmet sich Vf. Datierungsfragen und dem Sitz im Leben. Er kontrastiert die Interpretationen Wilckes und Fraynes, welche die Entstehungszeit der Hymnen $\mathrm{D}(+\mathrm{X})$ und $\mathrm{F}$ auf Grund textimmanenter Kriterien zu Beginn der Regierungszeit Šulgis ansetzen (Krönungshymne?), mit der Skepsis Veldhuis', der fragt, ob historische Anspielungen in literarischen Texten überhaupt zur Datierung dieser Werke herangezogen werden dürfen (S. 17-19). Da mit einer Ausnahme alle Šulgi-Hymnen nur aB bezeugt sind, scheinen Abfassungszeit und Überlieferungshorizont nicht übereinzustimmen (so die communis opinio), doch will Vf. kein abschließendes Fazit ziehen: eine anfängliche orale Tradition durch Sänger, die erst in aB Zeit verschriftet wird oder eine wenigstens partielle zeitgenössische Veröffentlichung von Hymnen auf Stelen, die später auf Tontafeln abgeschrieben wurden, hält er ebenso möglich wie eine Abfassung der Lieder in der aB Schule (S. 20-22). Die Diskussion schließt mit knappen Bemerkungen zu archaischen (oder archaisierenden) Schreibungen, die Relikte der Ur III-Orthographie darzustellen scheinen. 
Beispiele aus Šulgi F finden sich in einer Tabelle zusammengestellt; für Ergänzungen vgl. die Rezension von J. Bauer in OrNS 83 (2014) S. 266.

Kapitel II stellt die einzelnen Textzeugen vor, die bis auf eine Ausnahme alle aus Nippur stammen, aber unterschiedliche Formate haben. Für die fragmentarisch erhaltenen mehrkolumnigen Tafeln C, E und G werden zudem gut durchdachte Rekonstruktionsversuche präsentiert. Die Tatsache, dass sich das Hauptmanuskript A (HS 1460) in der HilprechtSammlung befindet, rechtfertigt ferner die Aufnahme des Werks in die Reihe Texte und Materialien der Frau Professor Hilprecht Collection of Babylonian Antiquities im Eigentum der Friedrich-Schiller-Universität Jena, hrsg. vom damaligen Betreuer der Magisterarbeit, Prof. Dr. Manfred Krebernik.

Zur Umschrift in Kapitel III ist lediglich anzumerken, dass trotz der Aussage auf S. 37, die Lesungen seien den Konventionen der aBZL angepasst worden, dem Vf. einige „neue“ Lesungen entgangen sind:

S. 48 c.33: [sa4] $\rightarrow \mathrm{še}_{21}$ (aBZL 36; vgl. jedoch den einschränkenden Kommentar ad loc.)

S. 49 d.17, S. 52 e.31 et passim: $\left[\mathrm{mi}_{2}\right] \rightarrow \operatorname{mim} / \min _{2}$ (aBZL 450)

S. 51 e.15: $\left(\operatorname{tug}_{2}\right)$ besser tu 9 (aBZL 417)

S. 52 e.37: [šilig] $\rightarrow$ silig (aBZL 184)

S. 66 et passim: [ugu] $\rightarrow{ }^{\mathrm{u}} \mathrm{ugu}_{6}$ (aBZL 358)

S. 85 zu c.1-21 // G ii 1-23: [ $\left.\mathrm{sag}_{2}\right] \rightarrow{ }^{\mathrm{sag} 3}{ }^{\mathrm{saga}} \mathrm{7}$ (aBZL 143)

(Für einige Druckfehler vgl. bereits J. Bauer, OrNS 83 [2014] S. 269.)

In Anbetracht der teilweise komplizierten Überlieferungslage wäre ein rekonstruierter sumerischer Text, welcher der deutschen Übersetzung entspricht und zugleich die Zitierform der sumerischen Zeile festlegt, wünschenswert gewesen - zumal eine solche Präsentation des Textes (Partitur und textus receptus) inzwischen als Standard gelten kann. Vielleicht wäre dann auch bemerkt worden, dass in e.23ff. die Zuordnung von Manuskript E zu A in der Umschrift (S. 51) von jener in der Übersetzung (S. 60) abweicht.

Die auf den sumerischen Text folgende Übersetzung (Kapitel IV) ist sehr wörtlich gehalten. Während dies die Komplexität des Textes veranschaulicht, hätten gelegentlich etwas freiere, weniger ,sumerologische’ Formulierungen dem Lesefluss gut getan. 
Der Kommentar (Kapitel V) ist sehr gewissenhaft ausgeführt, obgleich man sich manchmal eine ausführlichere Diskussion der Grammatik bzw. der einzelnen Varianten gewünscht hätte. Ärgerlich sind jedoch die zahlreichen Verweise auf Sekundärliteratur ohne jegliche Erläuterung, die vom Leser verlangen, sämtliche Referenzen selbst noch einmal nachzuschlagen. Der Kommentar gleicht somit oft einem Zettelkasten, obwohl es dem Vf. ein Leichtes gewesen wäre, die von ihm akribisch zusammengetragenen Referenzen dem Leser knapp zusammenzufassen. Die häufigen Zitate von abweichenden Übersetzungen C. Wilckes sind jedoch ohne einen Kommentar, der das divergierende Verständnis des Vf. erklärt, überflüssig.

Wünschenswert hingegen wären Übersetzungen der zahlreichen Belegsammlungen im Kommentar (z. B. S. 66f. zu Z. 4-5, S. 75 zu Z. 45-46, S. 78 zu Z. 60, etc.) gewesen, zumal nicht immer nachvollziehbar ist, wann Belegstellen übersetzt werden und wann nicht. Im Falle des Zeilenkommentars zu Z. 60 kann der Leser beispielsweise ohne Übersetzungen und eine wenigstens knappe Auswertung der Belege für den meš-Baum in den publizierten ŠulgiHymnen wenig mit den Zitaten anfangen; eine Chance für eine vorläufige Untersuchung der Beziehung des Herrschers zu diesem Baum wurde vertan.

Besonders hilfreich wiederum ist die derzeit aktuellste Zusammenstellung insbesondere der Šulgi-Hymnen inklusive neu identifizierten Textvertretern in Kapitel VI. Lobenswert hervorzuheben ist auch der Index (Kapitel VIII), da er nicht nur in übersichtlicher Form die in Šulgi F belegten Personen, Ortsnamen und sumerischen Lemmata präsentiert, sondern auch deren deutsche Übersetzung sowie den Zeilenkontext mitliefert.

Abschließend noch einige Bemerkungen zu den Abbildungen (Kapitel IX). Für die neue Kopie des Vf. von HS 1460 (A) hätte man gut die ganze Seite ausnutzen können, zumal der Maßstab angegeben ist und auf S. 148 ein Größenvergleich zwischen Manuskript A und D in Echtgröße angestellt wird. Die Zeichen in den sehr dicht gepunkteten Passagen sind kaum noch zu erkennen, obwohl sie laut Foto in den meisten Fällen bei weitem nicht so zerstört sind wie die Kopie suggeriert (dies gilt z.B. für Z. 10 und 30 sowie mehrere Stellen auf der Rs. und dem oberen Rand). Kollationen des Vf. nach Fotos der Tafeln aus dem Archäologischen Museum Istanbul hätten dem Leser nachvollziehbar gemacht werden sollen. Bei den grundsätzlich guten Schwarz-Weiß-Fotos wären Zeilenangaben zur leichteren 
Orientierung des Lesers wünschenswert gewesen; außerdem hätte man den Tafelnummern die verwendeten Sigla beigeben sollen.

S. 4: Die deutsche Übersetzung des Refrains ist unnötig sperrig, zumal in Anm. 27 die ungewöhnliche bzw. , poetische' sumerische Syntax diskutiert wird und sie trotz Wortumstellungen im Deutschen die sumerische Wortfolge (ur-sag ama-ne 2 -e ur 5 -re baan-du ${ }_{2}{ }^{d}$ nin-sumun 2 -na-ke 4 ) nicht exakt wiedergibt: das hätte ,Den Helden, seine Mutter hat ihn dazu geboren, Ninsumuna“ gelautet. Weitaus verständlicher wäre: „Dazu hat seine Mutter, Ninsumuna, den Helden geboren.“ Die Hervorhebung der göttlichen Mutter bleibt auch so durch den für die sumerische Literatur typischen Übergang vom Abstrakten [„Mutter“] zum Konkreten [,Ninsumuna“] erhalten. Während Vf. hier zwar skrupulös, aber inkonsequent den sumerischen Text wiederzugeben versucht, fragt man sich wenig später, warum auf S. 8 die im Sumerischen jeweils gleichlautenden Zeilen 5' und 10' $\alpha$ der Hymne Sîn-iddinam A unterschiedlich übersetzt werden.

S. 12: tum 2 -mu-un-ze 2 -en ist eindeutig ein Imperativ Plural („Bringt!“).

S. 37f./55 zu Z. 6-19: Aus rein grammatikalischer Sicht ergeben sich für diese Zeilen zwei Abschnitte bzw. Hauptsätze: Die infiniten Verbalformen in Z. 7-9 (\{ed+e\} gefolgt von zwei „,pronominalen Konjugationen“ mit hamțu-Basen!) münden in zwei Prospektiven in Z. 10 und 11, auf welche in Z. 14 der obligatorische Hauptsatz, mit Nanna im Ergativ, folgt. Z. 15-19 vertauschen die Reihenfolge, indem sie mit einem Prospektiv in Z. 15 beginnen, worauf zwei weitere infinite Verbalformen in $\{\mathrm{ed}+\mathrm{e}\}$ eingeschoben werden, bevor alles in den finiten Verbalformen des Refrains (Z. 18-19) mündet. So gesehen ist Nanna Akteur in den Z. 6-14; in den Z. 15ff. wird dessen Handlung durch Šulgi (der nur indirekt als Akteur präsentiert wird) auf formaler wie inhaltlicher Ebene, gespiegelt'. Seine Opferhandlungen erhalten durch die Imitation des göttlichen Vorbilds ihre Legitimation.

S. 37/69 zu Z. 7: Die Schreibung enku ${ }_{\mathrm{x}}{ }^{\mathrm{ku}}{ }$ suggeriert einen Fischnamen ( ${ }^{\mathrm{ku} 6}$ als nachgestelltes Fisch-Determinativ). In aB Zeit ist HA allerdings obligatorischer Bestandteil von enku und wurde offenbar nicht mehr als Lautindikator wahrgenommen. Daher wäre es weniger missverständlich, den von aBZL 206 angesetzten Lautwert enku (ZAG.HA) zu verwenden. 
S. 38/55 zu Z. 10: Bei dem semantischen Kompositum si sa $a_{2}$ sollte akan als (postuliertes) semantisches Objekt eigentlich nicht im Absolutiv, sondern im Direktiv stehen, für amar wäre in dem Fall wohl der Terminativ zu erwarten. Auf C. Wilckes einschlägige Studie in ASJ 22 (2000/2005) 279-301 hätte gerade in Bezug auf die Neutralisation der inneren Rektion des Verbs verwiesen werden können.

S. 40/S. 56 zu Z. 44: ša 3 kur-kur-ra-ke 4 wäre eindeutiger als ,die Herzen aller Fremdländer" zu übersetzen.

S. 40/S. $56 \mathrm{zu}$ 45-47: us 2 -sa-ba in Z. 46 ist parallel zu g ar-ra-ba in Z. 45 konstruiert und daher ebenso keine finite Verbalform - die folgt erst in Z. 47. Übersetze: „Nachdem das Fremdland (auf seinen Platz) gestellt (und) fest gegründet war (und) Tage der Fülle das Land erreichten, da streckte die Himmelsgöttin ihre Brüste zur Erde hin aus.“

S. 40/77 mit Anm. 106 zu Z. 48-49: Ein mu-Präfix mit dem Meer als Agens erscheint ungewöhnlich; ebenso hätte das Terminativinfix in Verbindung mit der verbalen Basis (ki - -) taka4 eine Diskussion verdient (,übrig bleiben“ wird in „Enmerkara und Ensukukešdana“ 37, 68 und 111 im Gegensatz zu vorliegender Stelle in allen Manuskripten durchgängig mit dem Lokativ konstruiert!). Davon abgesehen ist eine übriggebliebene Rohrmatte schwerlich ein treffendes Bild für Überfluss.

S. 40/56 zu Z. 60: Im Sumerischen zwei parallele Hauptsätze; die Übersetzung des Vf. suggeriert etwas wie *a zal-le du ${ }_{11-g a}$. Zum (hier unerwarteten) mu-Präfix bei mu-du ${ }_{11} \mathrm{~s}$. ELS $\$ 177 a) 1^{\circ}$.

S. 41/S. 56 mit Anm. 13 zu Z. 63: Eine Bedeutung „Zeder“ für irina 3 ist meines Wissens nicht belegt (so auch J. Bauer, OrNS 83 [2014] S. 267); Verwechslung mit eren? Für die

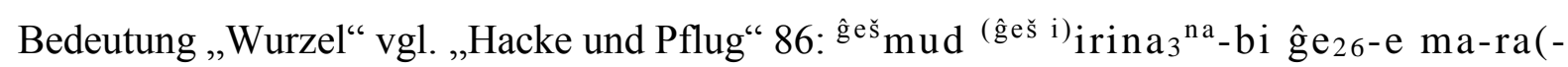
an)-de -de $_{5}$-ge(-en) ,Ich bin es, die Stämme und Wurzeln für dich einsammelt“ und 94:

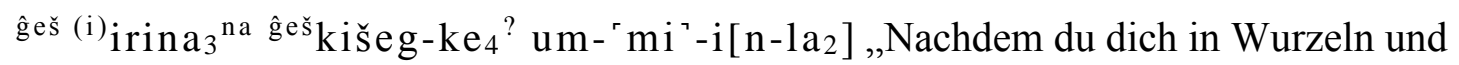
Dornengestrüpp verfangen hast, ...“ (zitiert nach C. Mittermayer, ,, Was sprach der eine zum anderen? “ Argumentationsformen in den sumerischen Rangstreitgesprächen, Habilitationsschrift Tübingen 2014). Es wäre also für F eine Genitivverbindung *irina 3 
meš $\breve{3}_{3}$ (ak) „Wurzeln des meš-Baums“ anzusetzen. Dies erübrigt sich jedoch, da laut Foto definitiv kein $\mathrm{DIM}_{3}$ vorliegt (zu schmal, waagerechte Keile nicht diagonal). Vielmehr sieht man klar sowohl die beiden parallelen waagerechten als auch den abschließenden senkrechten Keil von ĜIŠ; auch die Proportionen stimmen im Vergleich zu den igs. recht breitgezogenen Zeichen. Die vom Vf. als die für $\mathrm{DIM}_{3}$ charakteristischen Winkelhakenpaare interpretierten Spuren stellen entweder Reste eines getilgten Zeichens oder einfach Kratzer dar. F schreibt demnach wie $\mathrm{A}^{\hat{\mathrm{g} e s ̌}}$ meš 3 .

S. 41/56f./81 zu Z. 67-69: Bedenkt man die eindeutige marû-Basis na-ĝa $a_{2}-\hat{g}_{2}$ in Z. 68, erscheint na-du-du ,er geht wahrlich (fröhlich ans Werk?)“ in Z. 69 (Ms. A) gegenüber na$\mathrm{du}_{11}-\mathrm{du}_{11}$ in B als die bessere Variante. Somit enthielten die Z. 69-71 allesamt Bewegungsverben $\left(\mathrm{ku}_{4}, \mathrm{du}-\mathrm{du}\right.$, giri $\left.{ }_{3} \mathrm{dab}_{5}\right)$; die Variante in B scheint eine andere Rezeption des Sachverhalts darzustellen. Beachte in diesem Fall auch die vom Vf. nicht diskutierte Variante mu-na-an-ku 4 in $\mathrm{B}$, die möglicherweise (wenn /n/ kein Allomorph eines in dieser Zeile sonst nicht bezeugten Lokativs darstellt) auf ein transitives Verständnis „er brachte es zu ihm hinein“ deuten könnte.

S. 42/57 zu Z. 79: an-ĝa $a_{2}$-ar-ĝa $a_{2}$-ar bzw. ĝar-ĝar stellen eindeutig reduplizierte hamtuBasen dar; übersetze „der alle me an ihren Platz gestellt hat.“

S. 43 zu Z. 86 mit Anm. 30: Laut Kollation des Vf. (S. 146) ' ab `; die Übersetzung auf S. 57 wäre entsprechend anzupassen.

S. 46 zu G ii 10-11: Nach Foto: KA 'SUR x x [...]| 'UN? (x) ki-bala ${ }^{\top}[\ldots]$.

S. 48 zu c.36: Gegen die Ergänzung mit dem semantischen Kompositum šu - - du 7 spricht gegebenenfalls der Lokativ(?) nach nam-lugal, da es als semantisches Objekt des zusammengesetzten Verbs im Direktiv stehen sollte. Alternativ wäre auch ein regensloser Genitiv (,das des Königtums“") denkbar: „Das des Königtums [...] er mir in die Hand.“

S. 48/S. 59 zu d.3: Besser: „Ezinam (fehlender Ergativ nach Personennamen, vgl. ELS §138c) hat mit mir (= in meiner Gegenwart) Getreide in die Erde gefüllt.“ 
S. 49/S. 59 zu d.11-13: Vf. nimmt in d.11 (semantisch sinnvolle) antizipierte Genitive an, die jedoch nicht dastehen. In d.13 stellt die zweite Zeilenhälfte keinen Hauptsatz dar, sondern einen auf die ,pronominale Konjugation“ $\mathrm{e}_{3}$-a-ni folgenden Temporalsatz: ,,als ein gutes [Schicksal] entschieden wurde, [...].“

S. 50/S. 60 zu e.6: -bi-da ist hier wohl eher koordinierende Konjunktion als Komitativ: „Himmel und Erde [...].“

S. 51/S. 60 zu e.16: In/m Überfluss (/he ga

S. 53/S. 61 zu e.44: „Menschen“ sind im sumerischen Text nicht zu finden. tumu $\mathrm{u}_{18}\left(\right.$ ĜIŠGAL) $-1 \mathrm{u}=$ meḩû ist der Südwind; Verwechslung mit (nam-) $1 \mathrm{u}_{2}-1 \mathrm{u}_{7}(\hat{\mathrm{G} I S ̌ G A L})^{(1 \mathrm{u})}$ „Mensch(heit)“, konventionell (nam-) $1 \mathrm{u}_{2}-\mathrm{u}_{18}-1 \mathrm{u}$ umschrieben?

S. 54/61 zu e.59: di-da als subordiniertes marû-Partizip ist schwerlich „gesprochen wurde“, eher: ,die spricht“".

S. 54/61 zu e.65: za3-mim ist „Lob“, nicht „Lied“ ( $\left.\operatorname{ser}_{3}\right)$.

S. 76 zu Z. 47: Eine etwas ausführlichere Diskussion der „Brüste des Himmels“, die den Vf. zur Übersetzung Himmelsgöttin führen, wäre hilfreich gewesen. So zeigen die verhältnismäßig wenigen Belegstellen, dass sich die Vorstellung eines weiblichen Himmels ausschließlich in hymnischen Texten (Išme-Dagan D 25', Kusu A 33, Nanna L 24, Tempelhymne 332 [H 27]) zu finden scheint.

Insgesamt ist dem Vf. zu einer äußerst soliden Textedition zu gratulieren, welche dank gewissenhafter philologischer Arbeit und umfassender Kenntnis des Corpus der sumerischen Königshymnen eine verlässliche Grundlage für zukünftige Forschung bildet. 